\title{
The Case for gait analysis as part of the management of incomplete spinal cord injury
}

\author{
JH Patrick ${ }^{1}$ \\ ${ }^{1}$ Robert Jones \& Agnes Hunt Orthopaedic \& District Hospital NHS Trust, Oswestry, Shropshire, UK
}

Incomplete spinal cord injury at ASIA (D) level often leads to major gait re-education attempts for patients and staff. Walking prognosis usually depends upon muscle power loss, degree of spasticity present, type of lower limb joint deformity developing and availability of treatment. As an adjunct to physical examination and observation, instrumented gait analysis can inform treatment, since three-dimensional dynamic joint range motion (kinematics) and estimation of joint forces occurring at the hip, knee and especially ankle/foot (kinetics) can be combined with walking EMG and energy cost tests to understand fully a gait deficit. This should then assist the clinical decision-making process. Knowledge of the effects of any dynamic contracture on the available redundancy within ipsilateral limb joints, and information about muscle actions obtained by calculating joint forces (including moments and powers) improves overall knowledge of gait cycle abnormality. Such gait analysis has revolutionised the management of spasticity in walking cerebral palsy children by guiding surgical and other treatments. Similar improvements could be looked for in incomplete SCI patients whose gait pattern is not ideal. Dynamic energy cost estimations could also be used as an outcome measure to study improvements after treatment: whether this is orthotic, pharmacological or following physiotherapy or surgical techniques.

Spinal Cord (2003) 41, 479-482. doi:10.1038/sj.sc.3101524

Keywords: spinal cord injury (SCI); gait analysis; ASIA (D) paraplegia

\section{Introduction}

Advancing technology is credited with ever improving medical diagnosis and treatment, but the incomplete spinal cord injury (SCI) sufferer is, illogically, often left out of such investigative virulence. Recovery of some muscle and sensory activity becomes the province of the physiotherapist and the splint maker. Gait analysis techniques have not been shown as useful investigations for the assessment of such physical handicap. After neurological recovery begins, the need for computed tomography or magnetic resonance imaging (MRI) occurs only to look for a syrinx, and neurosurgical or orthopaedic treatment depends upon a series of such tests allied with quantified or definite evidence of neurological dysfunctioning. Walking is commonly affected in ASIA (D) ${ }^{1}$ lesions. We believe today that while no investigation, let alone practical treatment, deserves uncritical application to such persons, nevertheless they may be able to make more of their situation when the mandatory 2 years of available recovery have passed. Many patients at this stage have been 'lost to follow-up' or are not intensively reviewed. Technical

*Correspondence: JH Patrick, Robert Jones \& Agnes Hunt Orthopaedic Hospital, Oswestry, Shropshire SY10 7AG, UK dynamic studies of the gait (which may enable greater understanding of a physical problem) remain little used. Why is this so? Clearly, human gait depends upon the production of muscle activity to move the limb forward and these actions can be measured in a gait laboratory. Movement analysis is a clinical tool that allows increased understanding of the gait pattern in both normal and disease states, measuring spatial and temporal parameters, and also the kinematics (joint range changes) and kinetics (moments and forces acting across joints). Our knowledge of normal action of muscles to stabilise joints and empower dynamic activity is now understood and can be measured. ${ }^{2}$ We can discover the instinctive compensations for an impairment by employing gait analysis to assist the clinical opinion. In children, dramatic improvements have occurred in patients after surgical treatment, which has been preceded by 'gait analysis." $2-9$

\section{Why instrumented gait analysis?}

Gait laboratory investigations give scientific backing and explanation of the redundancy being used subsequent to injury, as compensation for neuromuscular 
inefficiency. We believe that greater understanding of a walking pattern can therefore direct a treatment intervention, or maybe, prevent time-consuming or expensive attempts at treatment of a handicap in those ASIA (D) ${ }^{1}$ patients whose continuing desire is 'to turn the clock back', but in whom the depth of impairment is too great, for success.

The surgeons of the last century were very aware of gait problems while treating the effects of poliomyelitis, tuberculosis and bony infection. These diseases have been replaced today by an overwhelming number of musculo-skeletal ageing problems. Joint replacement has preoccupied those treating gait handicap, to the exclusion of much else. However, walking problems post-ASIA (D) ${ }^{1}$ injury have led to a wave of interest in using medical paralysants like Botulinum toxin ${ }^{10,11}$ in combination with splinting, to alter gait patterns. We expect such gait pathology in SCI adults to be improved if gait assessment guides treatment.

\section{Accessibility}

A 2-hour gait analysis cost is comparable to that of MRI, but gait laboratories are few in number in comparison with imaging suites. Reasons for this include the results of the analysis being technical and perhaps nonunderstandable or irrelevant. Measurements of knee moments might be of interest to doctors and engineers designing joint replacements but is of no immediate benefit to rehabilitation physicians. Comments on muscle power imbalance at a joint could be part of the gait analysis report. Thus, clinical understanding can be improved provided that the information is as accessible to the doctor as are the anatomical pictures from MRI. Few of us completely understand the physics of MRI production, but we like the image!

Gait analysis needs to combine clinical need with the scientific foundation and evaluation methods used in science. ${ }^{12}$ Both should complement each other, and improved quality of measurement now allows the results of an intervention to be measured.

\section{Use of gait investigations}

What then can a gait laboratory do to investigate the poor gait of an incomplete paraplegic? It is important, obviously, to have the right equipment and for the clinician to be able to depend upon the quality standards of the gait laboratory. However, the most important feature - as always in medicine - is the defining of objectives.

A rehabilitationist doctor understands the importance of intact nerve and muscular function. Loss of joint range (a contracture) is obvious but may be more difficult to diagnose if it is only 'mild' OR that it is only seen dynamically - as part of the walking problem in the lower limb in the neurologically impaired. Couch examination may show normal passive ranges when the examiner pushes 'hard'. We all try, for example, to convince ourselves that a slight loss of ankle dorsiflexion occurring when the knee is straightened is not an equinus deformity. We may be fooling ourselves as often such patients will show a lack of foot clearance during the swing phase of gait. The possible explanations for this may a partial or complete paralysis of the ankle extensors, or some degree of spasticity of the ankle plantarflexors. This is known to all of us and muscle imbalance at a joint is supposedly an easy clinical judgement, but the complication of a dynamic control problem is easily overlooked. We believe that this is often seen in SCI patients during supine examination where only mild problems are found, but the difficulty is revealed during walking. A dynamic technological measure using accurate three-dimensional analysis and modern technology ${ }^{13}$ is better than a guess or 'clinical experience/intuition', in such cases. There are many walking patients where the observers' eye is tricked (after all the human eye can only see 12 frames/s; our laboratory cameras, at least 50). More exact examples include an inappropriate action of the rectus femoris muscle in swing phase preventing knee flexion, through its attachment distally to the tibia. This 'lengthens' the limb during swing and produces shoe scuffing or a need for circumduction of the leg. A dynamic rectus EMG should show this, providing muscle firing information and thus suggesting treatment. Equally, the methods used by the patient to overcome hip weakness may not be apparent by conventional examination, but dynamically may be shown as the cause of the toe scuffing (the hip flexor main accelerator for swing-phase advancement of the limb, if weak, cannot effectively lift the foot off the floor in initial swing phase). Opposite-side hip abductor weakness may also allow the swing-side hemipelvis to 'drop', producing foot/shoe friction at the floor. Gait laboratory kinematic (joint range motion) and kinetics (estimation of joint forces and moments) should help to identify the cause. Treatment of this excess shoe wear might then be directed proximally in the limb, rather than us registering complaints about the AFO to the orthorise.

The most common example occurs in cases of equinus. Traditionally, we may appreciate that a severe toe-walk is a useful compensation for weak knee extensors in a boy with Duchenne muscular dystrophy. His gait pattern keeps the knee straight by bringing the ground reaction vector in front of the knee, 'straightening' it. Lengthening of the heel cord 'to bring the heel down' is known to be disastrous since the reaction point of the body weight is surgically changed after lengthening. ${ }^{14}$ With the heel on the ground, the ground reaction vector of the body weight will fall behind the knee centre, and as a result walking will cease since the quadricep muscle 'power' is too small to counter the knee flexion moment. The knee then stays flexed and the effort required to stand is excessive, so ambulation ceases. Similar situations may occur after SCI, although such quadriceps weakness is not so common. Targeted physiotherapy treatment can be directed to strengthening muscle directly. In most SCI patients, a spastic calf musculature with a similar visible equinus may profit by 
heel-cord lengthening to bring the ground reaction force within the confines of the knee joint, rather than grossly in front of it. A clinical clue of this is dynamic recurvatum (hyperextension of the knee in stance phase). Surgical treatment is advised to change the ground reaction vector (grv), so that it lines up (as near as possible) with all the joint centres in the lower limb if so, the walk will improve and the energy cost will lessen. However, results are variable because the surgical objective in such spasticity states may fail. If the patient already walks with bent knees and ankle tendon lengthening is performed, then the surgery may shift the grv behind the knee joint centres - worsening the 'crouch' position. This distinction can be made by an experienced clinician, or be confirmed in a gait laboratory. Assessing the position of the grv allows knowledgeable prediction of outcome should surgical treatment be debated, whatever the aetiology of the neurological handicap. Decision-making is improved when a gait laboratory force-platform is used. ${ }^{15}$ Biomechanical understanding prevents the undertaking of inappropriate surgery, or ineffectual and expensive treatment with Botulinum toxin. Correct surgery should realign the grv during walking and thus reduce energy consumption during gait. The possibility of motor learning ${ }^{16}$ using leg orthoses to normalise biomechanics is also a possibility.

\section{Proof - suggested evidence}

We make reference here to the modern treatment of diplegic cerebral palsy. ${ }^{17-19}$ All have shown that the poorly cosmetic, dynamically exhausting, gait pattern of cerebral palsy children can be improved by use of a gait analysis to guide the surgeons knife or the Botulinum injection and splint usage (below knee orthosis). Identification of dynamic gait abnormality and distinction of primary cause from secondary effect is relatively easy in cerebral palsy using kinematics and especially kinetic analysis measurement. There is no reason why the same distinction cannot be made in SCI spasticity or part paralysis in adults whose problems are caused by a different insult, but whose lower limb biomechanical defects are often analogous.

However, surgical or other changes of one element of the gait can produce somewhat unpredictable outcomes in adult cerebral palsy patients as is often stated by known experts. ${ }^{20}$ This may stay our hand, when considering management of the adult SCI patient, especially given our poor understanding of the interrelationships between each orthogonal plane (sagittal, transverse and coronal), together with age and weight (mass) changes. Governance pressure today provokes us to be critical of treatments, and always we need to consider outcome measurement for various treatments in different patient groups. The effects of lessening spasticity at lower limb joint levels, using Botulinum injections, is fast opening up opportunities to try out different treatments, but patient and carer expectations may be dashed if no measured effect or defining of treatment protocols exist, and poor long-term results occur.

All interventions should be measured against available results. At present, highly accurate, validated and repeatable measures can be taken prior to, and after physiotherapy, splinting or Botulinum toxin treatment. Particularly, energy consumption during walking has been refined. A specific test, the 'oxygen cost' test (millilitres of $\mathrm{O}_{2}$ consumed $/ \mathrm{min} / \mathrm{kg} /$ metre walked), (COSMED Via dei Pianide Monte, Savello 37, Pavona di Albano, Rome 1-00040) can be used on SCI patients to measure accurately the energy requirement in walking by breath-by-breath gas analysis. This is a major investigative tool now available in the gait laboratory environment. A lowered oxygen cost of walking is likely proof that an intervention has worked.

Such measurement should provide evidence-based results to allow comparisons and research between different treatment protocols and centres.

Adult neurological disease often presents a dynamic management puzzle. Concern is felt about offering orthotic or surgical treatment for walking impairments, when the outcome of one treatment modality alone is unknown. The degree of redundancy that exists in the neuromuscular system is large and sometimes unappreciated by clinicians. Instead of performing the intervention, newer methods of mathematical modelling (by Software for Interactive Musculo-skeletal Modelling $(\mathrm{SIMM}))^{21}$ and some applications of forward dynamic analysis allow simulation of the results of a procedure 'on the computer' We are beginning to become predictive. Measures to plan gait re-education may become very worthwhile. These methods depend upon gait laboratory findings, and although research tools at present show some promise for the future.

Less esoteric tests include dynamic lower limb EMG recordings. They can intensify diagnostic purity and thereby assist treatment by confirming muscle activity or its absence during the gait cycle. In clinically confusing cases, evaluation of dynamic EMG and the joint kinematics and powers can allow an insight into how observed effects occur in pathological gait. To discover an inappropriately active muscle firing at the wrong time in the gait cycle, or one that is 'silent' when it should be active, can help confirm a diagnosis or explain a deficiency; unbalanced paralysis at a joint can lead to curious deformity and a puzzle about the cause. We have been able to confirm cases of suspected hysteria, or the Munchausen syndrome in similar circumstances.

Use of a gait laboratory in modern-day rehabilitation to follow the results of treatment is occurring sporadically in the developed countries - its future is bright as an educative tool, as well as a guide to the management of adult neuropathological gait abnormality. Using careful gait analysis is worthwhile if it leads us to choose suitable cases for treatment, where we can 'expect' a given result: in some patients, we have recommended 'to leave well alone' because computer assists show us that we are observing a 'compensation' not a primary gait fault. As Gage, noted in the 
treatment of cerebral palsy spasticity, much opprobrium is attached to a treatment which grossly alters a compensation, but benefit occurs when a primary mobility problem is successfully treated. Effecting a mobility advantage for incomplete paraplegic patients is as important for them as it is for children. Cerebral palsy children have gained significant improvement in the last 15 years by gait assessment prior to treatment using surgical, orthotic or physiotherapy interventions. Although agreement on studying a suitably homogeneous group of SCI patients might be difficult, we feel that their walking efficiency can be improved. Since $86 \%$ of patients with incomplete tetraplegia will recover ambulatory function to some extent, ${ }^{22}$ the rehabilitationist needs to consider how to assess patients better using clinical examination and judgement, the WISCI scales, ${ }^{23,24}$ and, we believe, gait laboratory help to maximise patient benefit.

\section{References}

1 Maynard Jr FM et al. International standards for neurology and functional classifications of spinal cord injury. Spinal Cord 1997; 35: 266-274.

2 Jonkers I, Stewart C, Spaepen A. The study of muscle action during single support and swing phase of gait: clinical relevance of forward stimulation techniques. Gait Posture 2003; 17: 97-105.

3 Sutherland DH, Hagys JH. Measurement of gait movements from motion picture film. J Bone Joint Surg 1972; 54A: 788-789.

4 Gage JR. Gait analysis for decision making in cerebral palsy. Bull Hasp Joint Dis Orth Inst 1983; 43: 147-163.

5 Perry J. Distal rectus femoris transfer. Dev Med Child Neurol 1987; 29: 153-158.

6 Patrick JH. Cerebral palsy diplegia; improvements for walking. BMJ 1989; 299: 1115-1116.

7 Patrick JH. Use of movement analysis in understanding gait abnormalities in cerebral palsy. Arch Dis Childhood 1991; 66: 900-903.

8 Nene AV, Evans GA, Patrick JH. Simultaneous multiple operations for spastic diplegia - outcome and functional assessment of walking in 18 patients. J Bone Joint Surg 1993; 75B: 488-494.
9 Gage JA. Gait analysis; An essential tool in the management of cerebral palsy. Clin Orthop Related Res 1993; 288: $126-134$.

10 Al-Khodairy AT, Gobelet C, Rossier AB. Has botulinum toxin type A a place in the treatment of spasticity in spinal cord injury patients? Spinal Cord 1998; 36: 854-858.

11 Burchiel KJ, Hsu FPK. Pain and spasticity after spinal cord injury. Spine 2001; 26 (Suppl): 154.

12 Kleissen RFM. Factors in clinical application of gait analysis. J Rehabil Sci 1990; 3: 106-109.

13 Whittle MW. Gait Analysis. An Introduction. Oxford: Butterworth-Heinemann, 1996.

14 Khodadadeh S et al. Knee moments in Duchenne muscular dystrophy. Lancet 1986; 544-545.

15 Ounpuu S, Gage JR, Davies RB. Three dimensional lower extremity joint kinetics in normal paediatric gait. $J$ Paediatr Orthop 1991; 11: 341-439.

16 Butler PB, Thompson N, Major RM. Improvement in walking performance of children with cerebral palsy: preliminary results. Dev Med Child Neurol 1992; 34: 567-576.

17 Sutherland DH et al. Double blind study of Botulinum A toxin injections into the gastrocnemius muscle in patients with cerebral palsy. Gait Posture 1999; 10: 1-9.

18 Gage JR. Gait Analysis in Cerebral Palsy. Mac Keith Press 1991.

19 Vaughan CL, Damiano DL, Abel MF. Gait of normal children and those with cerebral palsy. In: Allard P, Capozzo A, Lundberg A, Vaughan C (eds). Three Dimensional Analysis of Human Locomotion Wiley: John Wiley \& Sons Ltd 1997, pp 335-361.

20 Doederlein L. 'Gait analysis and adult cerebral palsy surgery'. CP Surgery Society Meeting, Heidelberg, 1998.

21 Delp SL et al. An interactive graphics-based model of the lower extremity to study orthopaedic surgical procedures. IEEE Trans Biomed Eng 1990; 37: 757-767.

22 Burns SP et al. Recovery of ambulation in motorincomplete tetraplegia. Arch Phys Med Rehabil 1997; 78: 1169-1172.

23 Ditunno JF et al. Walking index for spinal cord injury (WISCI): an international multi-centre validity and reliability study. Spinal Cord 2000; 39: 234-243.

24 Ditunno PL, Ditunno Jr JF. Walking index for spinal cord injury (WICSI II): scale revision. Spinal Cord 2001; 39: 654-656. 